\title{
BMJ Open Quality Improving paediatric flow in an UK Paediatric Assessment Unit
}

Danning Li, Movin Abeywickrema, Sharvari Vadeyar, Abigail Ward, Thomas Abberton, Justina Rweyemamu

To cite: Li D, Abeywickrema M, Vadeyar S, et al. Improving paediatric flow in an UK Paediatric Assessment Unit. BMJ Open Quality 2022;11:e001561. doi:10.1136/ bmjoq-2021-001561

Received 8 June 2021 Accepted 13 January 2022

\section{Check for updates}

(C) Author(s) (or their employer(s)) 2022. Re-use permitted under CC BY-NC. No commercial re-use. See rights and permissions. Published by BMJ.

Department of Paediatrics, Birmingham Heartlands Hospital, Birmingham, UK

Correspondence to

Dr Danning Li;

danning.li@nhs.net

\section{ABSTRACT}

The 2010 Royal College of Paediatrics and Child Health (RCPCH) guidelines for acute paediatric services set standards for time to senior review for paediatric medical admissions in the UK as tier two doctor (registrar) review within 4 hours and consultant review within 14 hours. Our aim was to implement these standards in our unit through increasing proportions of reviews within these timeframes and measuring the impact on patient flow. Four quality improvement cycles were completed between March 2018 and March 2020 capturing data from 288 patient data sets. Recommendations included the extension of consultant on-site availability out of routine working hours (after cycle 1), highlighting patients awaiting consultant review during team handover (after cycle 2), and improving tier two doctor rostering (after cycle 3). After highlighting patients for consultant priority review, the proportion of patients seen within 14 hours improved from $53.3 \%$ (cycle 2) to $95 \%$ (cycle 3, $p=0.005$ ). Improved tier two doctor cover increased the proportion meeting registrar review within 4 hours from $82.9 \%$ (cycle 3 ) to $96.2 \%$ (cycle $4, p=0.028$ ). A large proportion of paediatric patients were managed and discharged at tier two doctor level (65.6\% over cycles 1-4). An inverse correlation was seen $(R=-0.587)$ between time to discharge and the number of tier two doctors on shift (cycle 4). The interventions conducted demonstrated significant improvement in proportions of paediatric patients seen within the $\mathrm{RCPCH}$ timeframes. Adequate tier two doctor staffing is a priority for prompt review and discharge of acute paediatric patients. Future work aims to consider factors such as nursing rostering, bed management and the impact of COVID-19 on paediatric flow.

\section{INTRODUCTION}

\section{Problem description}

Birmingham Heartlands Hospital is a busy teaching hospital with a large paediatric department, which serves a diverse patient demographic and receives a wide range of acute admissions. The department is staffed by a minimum of one on-call consultant, one tier two doctor and one tier one doctor. This project focused on the acute admissions to this Paediatric Assessment Unit (PAU). Emergency department referrals are transferred to the PAU, after which patients are admitted as inpatients, or discharged with or without a subsequent observation period.
Five quality improvement cycles implementing 'Facing the Future: standards for acute paediatric services' were completed, comparing paediatric flow in Birmingham Heartlands Hospital against these standards and analysing the multifactorial contributors affecting prompt patient review.

\section{Available knowledge}

Increased waiting times in UK emergency departments have been associated with poorer outcomes for patients including longer length of stay and increased morbidity and mortality. ${ }^{1}$ Coroner's reports were highlighted to the Royal College of Paediatrics and Child Health (RCPCH) in 2014, which identified a higher mortality in children with longer waiting times. ${ }^{2}$ The findings from audits conducted in 2013 and 2017 by RCPCH found that acute paediatric services were not meeting the national standards outlined in Facing the Future: standards for acute general paediatric services. ${ }^{3}$ This indicates that there is an urgent need to improve acute paediatric services in line with RCPCH guidance.

\section{Rationale}

In Facing the Future: standards for acute general paediatric services, RCPCH outlines that all acute paediatric admissions should be seen by a tier two (middle grade) doctor within 4 hours of admission and by a consultant paediatrician within 14 hours of admission. ${ }^{2}$

Junior doctor staging in this quality improvement project have been defined as per the Facing the Future standards. Tier one doctors refer to doctors within foundation years (F1/F2 doctors) and in earlier paediatric training (Specialty Trainee years 1-3, also known as Senior House Officers) whereas tier two doctors have been defined as those equivalent to higher paediatric trainees (Specialty Trainee years 4-8, also known as Specialist Registrars, ST4-8). These doctors lead out-of-hours paediatric admissions, and hence manage flow through paediatric 
admissions pathways especially outside of non-office hours, making recording time to be reviewed by these doctors an important measurement. Tier three doctors have been referred to as paediatric consultants.

\section{Specific aims}

Our SMART aim was to reduce the time from admission to being seen as per Facing the Future guidelines-we aimed to increase the percentage of PAU admissions seen by a tier two doctor within 4 hours and a consultant by 14 hours by $5 \%$ per cycle, to $100 \%$ by end of Quality Improvement Project. We undertook four plan, do, study and act (PDSA) cycles to identify factors influencing paediatric flow through the department and therefore patient outcomes.

\section{METHODS}

\section{Context}

There are very few studies in the literature comparing departmental waiting times to the Facing the Future standards; however, a study conducted in Glasgow in 2018 found that time to review was a factor reducing a regional paediatric hospital's achievement of these standards. Recommendations provided from this study included increasing on-site consultant cover and redistributing senior doctors to areas such as the emergency department. ${ }^{4}$ The impact of this change is yet to be disseminated; however, this recommendation is reflected in one such change made following cycle 2 of this project.

Additionally, a meta-analysis of paediatric outcomes and admission times across the USA, UK, Australia, Kenya, Nigeria, the Netherlands and India across intervals between 1988 and 2015 found that there was a longer length of stay in acute paediatric admissions conducted over the weekend. The study discussed that while it was unclear whether or not staffing levels had an impact on this outcome, concerns regarding paediatric clinical staffing in the UK have been ongoing for the past few years. This meta-analysis also found an increase in patient complications over the weekend in certain conditions. ${ }^{5}$ These findings relate to the problem being tackled by this project through highlighting inequalities in paediatric outcomes for acute admissions which may be related to staffing levels, a factor which is explored through the interventions implemented through the PDSA cycles of this project.

Separate literature reviews of PubMed and Google Scholar using the search terms "paediatric* OR pediatric* AND (wait* OR "flow") AND quality AND improv* AND intervention*", and the search terms "paediatric* OR pediatric* AND wait* AND quality AND improv* AND intervention*" of English language studies published in the past 5 years. From this search conducted, it was apparent that little to no UK-based studies existed in recent times which sought to improve the issue of paediatric flow and waiting times.
Through the baseline measurement, it was evident that there was a need to gather further data relating to time to consultant review and also to devise strategies to improve compliance with standards. The team conducting this project were a series of junior doctors working in the hospital under the guidance of a consultant paediatrician with input from the audit department and audit lead of the paediatric department.

\section{INTERVENTIONS AND STUDY OF INTERVENTIONS Baseline measurement}

For this project, baseline measurement was collected during the trial period 15 March 2018-1 April 2018 in order to establish a baseline for further analysis. Clinical encounters of 36 randomly selected paediatric patients who attended the general paediatrics admissions unit during this time period were lifted from proformas stapled to the front of patient notes. Staff were asked to fill out the following balancing measures on the proforma: time of admission and time of nursing triage; as well as the following outcomes: time seen by any doctor, time seen by any senior (senior doctor defined as tier two or three level), and time of discharge.

Proformas were trialled to simplify data collection and minimise later time spent physically reviewing paper notes. Proformas were filled out by the nursing team and medical team on nursing triage and medical review, respectively. Times were documented on the proforma and within the actual notes themselves at the same time by the healthcare professional caring for the patient; these data were later verified by the lead clinician collecting the data for this cycle.

Proformas were poorly completed by staff members; from 36 proformas, 26 data sets were complete. Staff feedback stated that proformas were cumbersome and added to ongoing clinical duties. These proformas were not used for the following PDSA cycles.

\section{PDSA cycle 1: 20 March 2019-7 June 2019}

As of cycle 1, an updated version of the Facing the Future guideline also included a novel standard for time to consultant review of patients (all patients to be seen within 14 hours). As a result, cycle 1, unlike the baseline measurement, also included the outcome measure of time until consultant doctor review.

During this audit cycle, consultants were present from 09:00 to 21:00 on weekdays and 09:00 to 12:00 on weekends. It was noted that patients arriving after consultants had left for the day had prolonged waiting times to be seen as well as longer times until discharge. These patients would often not be seen by a consultant until ward round the next morning, and this effect was particularly pronounced in patients arriving after midday on weekends, as they could potentially face a wait of 19 hours before being seen on the 09:00 ward round the next day. The results of this cycle were used to fund the addition 


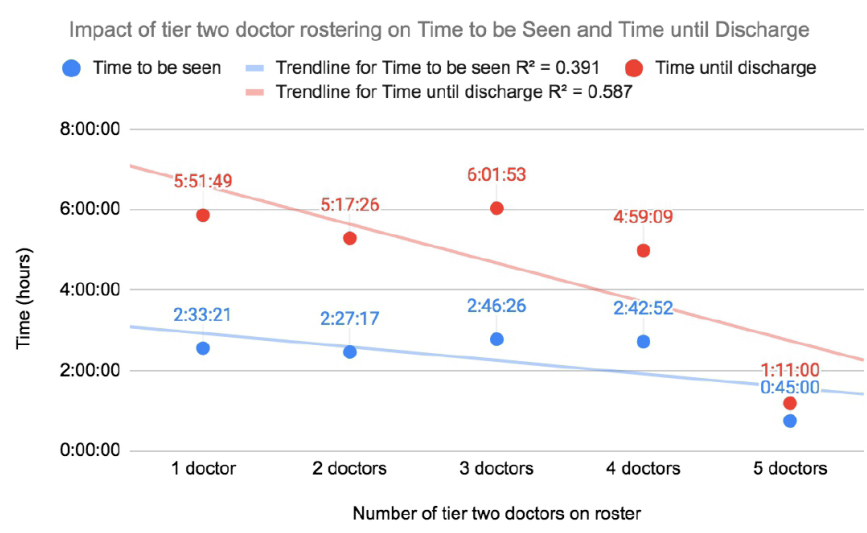

Figure 1 Impact of registrar rostering on time to be seen by a registrar doctor and time until discharge.

of three new consultants to the ward as well as extend consultant working hours on weekends.

\section{PDSA cycle 2: 1 August 2019-8 August 2019}

From July, consultant cover was extended over weekends from 09:00-12:00 to 09:00-17:00.

\section{PDSA cycle 3: 11 0ctober 2019-18 0ctober 2019}

Multiple changes were made after this PDSA cycle. Patients arriving after 17:00, when consultants had left site for the day, were marked with 'new' on the handover sheet. These patients would be preferentially seen by the consultant in the morning ward round, instead of being reviewed by a tier two doctor.

\section{PDSA Cycle 4: 1 March 2020-25 March 2020}

For our final test cycle, we analysed the impact of a novel tier two doctor roster on paediatric flow. It had been noted in cycle 3 that there was an uneven distribution of tier two doctors rostered throughout the week. Some days had five doctors rostered on, whereas others only had one.

As shown in figure 1, changes were made to the tier two doctor roster to ensure a minimum of two tier two doctors rostered on at all times. This was hugely successful, with the proportions seen by a tier two doctor as per Facing the Future guidelines increasing from $82.9 \%$ to $96.2 \%$.

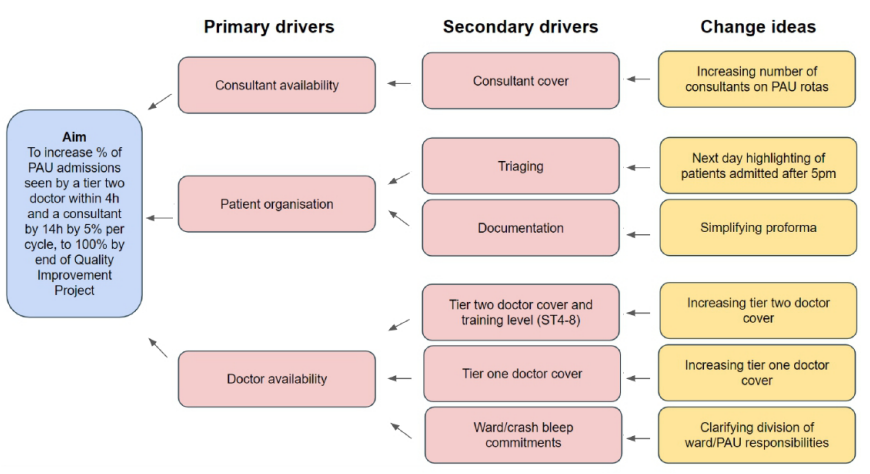

Figure 2 Driver diagram of Paediatric Assessment Unit (PAU) flow, and cycle interventions.

\section{Measures}

As shown in figure 2, the primary drivers contributing to paediatric flow were: tier three availability (number and presence of tier three paediatric doctors on-site), patient organisation through nursing and auxiliary team management (time of paediatric patient arrival, time until nursing triage), and tier one and tier two doctor availability.

In order to further understand the way in which these factors influenced the achievement of Facing the Future standards, a number of process measures were analysed, including: time to be seen by a doctor (of any grade), time to be seen by a tier two doctor, time to be seen by a tier three doctor and total time in PAU. Further balancing measures reported included: time of paediatric patient arrival, time until nursing triage and numbers of tier two and tier three doctors on-site.

Our main outcome measure was increasing the proportions of patients seen within the 4 and 14 hours window by a tier two doctor and a consultant-level doctor, as per Facing the Future guidelines.

\section{Analysis}

A retrospective analysis of paper patient notes was conducted after identifying admissions through electronic patient record systems. Sixty-four data sets were selected at random from the admissions to PAU for the dates corresponding to the PDSA cycle. Data were collected in order to gather information about the above factors and analysed using a spreadsheet software. After each cycle, different interventional strategies aimed at improving paediatric flow were developed from analysis of the results obtained and correlation with the primary drivers identified.

The interventions included the extension of consultant on-call cover (after cycle 1), changes in handover to highlight patients who had not had consultant review (after cycle 2), and roster changes with an increased number of rostered tier two doctors (after cycle 3). Following each intervention, the effects of this change were audited with the subsequent cycle in order to ascertain the impact on paediatric flow.

\section{RESULTS}

\section{Baseline measurement}

Of the data collected from the proformas, the following was complete: $100 \%$ of patient arrival time, $86.1 \%$ of time to be seen by any doctor, $97.2 \%$ of time to be seen by a senior, and $80.6 \%$ of time until discharge.

Incomplete data sets were excluded from this analysis, and only 26 data sets were used in this analysis. Time to consultant review was not documented in the baseline measurement, as this was only added to the revised Facing the Future guidelines in June 2018. All subsequent cycles contained time until consultant review.

Ninety-two per cent of paediatric patients (24 out of 26) were seen by a registrar or above within 4 hours, with the 
average wait time being $104.2 \mathrm{~min}$ (1 hour and $44 \mathrm{~min}$.) During this time period, 478 patients were seen on PAU, an average of 239 patients per week.

The national average of paediatric patients being seen by a middle grade or above in 4 hours was $79 \%$ in 2017 , and for those by a consultant $48 \%$. On average, our unit's performance is far higher than the national average.

The average time until discharge was $229.5 \mathrm{~min}$ ( 3 hours and $49.5 \mathrm{~min}$ ). There was no correlation between time to be seen by a doctor or total time in PAU in this data set. Time to consultant review was not documented in this PDSA cycle, as this was only added to the revised Facing the Future guidelines in June 2018. All subsequent cycles contained time until consultant review.

\section{PDSA cycle 1: 20 March 2019-7 June 2019}

There was a small decline to $83 \%$ of patients being by a tier two doctor or above within 4 hours. The average wait time was $104.2 \mathrm{~min}$ ( 1 hour and $44 \mathrm{~min}$ ). During this time period, 478 patients were seen on PAU, an average of 239 patients per week.

The average length of stay was 229.5 min ( 3 hours and $49.5 \mathrm{~min})$. There was no correlation between time to be seen by a doctor or total time in PAU in this data set.

As of cycle 1, an updated version of the Facing the Future guideline also included a novel standard for time to consultant review of patients (all patients to be seen within 14 hours). As a result, cycle 1, unlike the baseline measurement, also included measurements of time until consultant doctor review; in cycle $1,57.9 \%$ of patients were seen by a consultant within the 14-hour timeframe.

\section{PDSA cycle 2: 1 August 2019-8 August 2019}

From July, consultant cover was extended over weekends from 09:00-12:00 to 09:00-17:00.

Despite this, paediatric patient review as per Facing the Future guidelines did not improve. Only $79 \%$ of patients were seen by a tier two doctor or above within 4 hours, and $53.3 \%$ seen by a consultant within 14 hours.

Increasing tier two doctor cover correlated with increased proportions of patients seen within the 4 hours time limit. With increasing tier two doctor cover, $69.6 \%$ (one tier two doctor), $86.4 \%$ (two tier two doctors) and $100 \%$ (three tier two doctors) were seen, respectively, within 4 hours.

Analysis of non-admitted paediatric patients (defined as stay less than 24 hours) revealed a negative correlation between the time seen and total time in department with an $R$ value of 0.34 .

\section{PDSA cycle 3: 11 0ctober 2019-18 0ctober 2019}

The preferential review of patients arriving after 17:00 on the ward round the following morning by a consultant decreased the number of patients who stayed for longer than 14 hours without having consultant input.

Of the 19 patients seen by a consultant, 95\% (18 patients) were seen within a 14-hour timeframe.

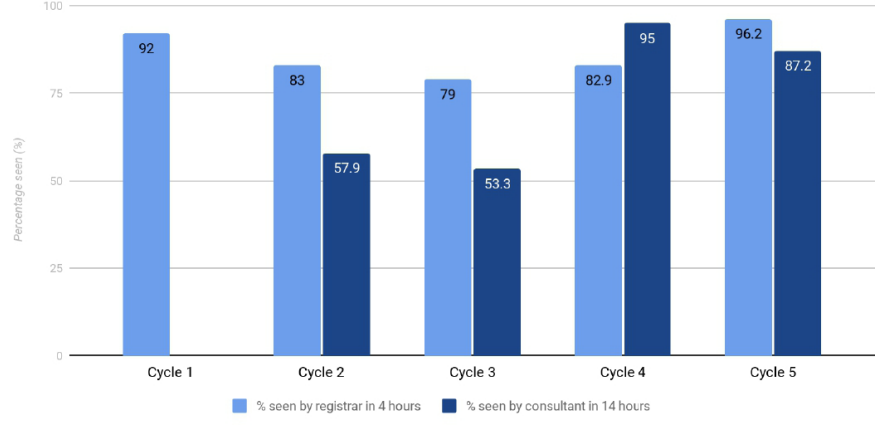

Figure 3 Proportions of patients reviewed within the Facing the Future standards across five plan, do, study and act cycles.

Again the correlation between time of being reviewed by the tier two doctor and the time until discharge has been analysed, both showing a negative correlation. The $\mathrm{R}$ values for this are 0.391 and 0.587 , respectively. No such correlation was demonstrated for consultant review and time until discharge.

\section{PDSA cycle 4: 1 March 2020-25 March 2020}

Changes made to the tier two doctor roster were hugely successful, with the proportions seen by a tier two doctor as per Facing the Future guidelines increasing from $82.9 \%$ to $96.2 \%$.

\section{Summary of results}

Our main outcome measure was increasing the proportions of patients seen within the 4 and 14 hours window by a tier two doctor and a consultant-level doctor, as per Facing the Future guidelines.

As shown in figure 3, extending consultant on-site cover altered the proportion of patients seen within 14 hours from $57.9 \%$ (cycle 1) to $53.3 \%$ (cycle 2, p=0.79) with improvement to $95 \%$ (cycle $3, p=0.005$ ) after highlighting consultant priority review.

Improved tier two doctor cover increased the proportion meeting senior review within 4 hours from $82.9 \%$ (cycle 3) to $96.2 \%$ (cycle $4, \mathrm{p}=0.028$ ).

A majority of paediatric patients were managed and discharged by a tier two level doctor $(65.6 \%$ over cycles 1-4). An inverse correlation was seen (R-value of -0.587) between time to final discharge and an increasing number of tier two doctors per shift (cycle 3). Consultant expansion showed an inverse correlation with time to consultant review (R-value -0.791 ).

Data from cycles 3 and 4 were analysed for increasing grade of tier two doctors (ST3-ST8) and average time until discharge. There was a weak positive correlation which was not statistically significant $(R=0.218, p=0.256)$.

Over time, the average length of stay increased from $229 \mathrm{~min}$ (baseline) to $258 \mathrm{~min}$ (cycle 1 ), to $318 \mathrm{~min}$ (cycle 2), to $334 \mathrm{~min}$ (cycle 3) and $329 \mathrm{~min}$ (cycle 4), despite earlier tier two and consultant review.

In the baseline measurement, there were 239 patients attending PAU on average per week, which has steadily fallen to 167 patients (cycle 1), 123 patients (cycle 2), 130 
patients (cycle 3) and 98 patients per week in the final PDSA cycle. As PAU admissions consist of GP (general practice) and emergency department referrals, this may be due to reduced referrals and increased paediatric presence.

\section{DISCUSSION}

\section{Summary}

We investigated the impact of extension of consultant on-site cover, changes to prioritisation for consultant review by waiting time and increases in tier two doctor rostering on the proportion of paediatric admissions that met the RCPCH Facing the Future standards. ${ }^{2}$

Increases in tier two doctor cover resulted in marked increases in proportions of patients seen by a tier two doctor within the 4 hours target (figure 1). As outlined in the results, the majority of patients being managed and discharged by a tier two doctor suggest that this cohort has a significant impact on patient flow through PAU. Additionally, the inverse correlation between the number of tier two doctors on roster and the time until discharge supports this inference (figure 1). Therefore, we argue that increasing tier two doctor cover is a primary priority in improving patient flow through PAU.

\section{Interpretation}

Although increasing consultant weekend cover contributed to increased proportions of patients seen within the Facing The Future 14 hours consultant target time window, it was improved prioritisation of these patients that had the single most significant impact (from 53.3\% to $95 \%$ of patients seen within the target) (figure 3). These findings support our understanding that with regards to roster planning, it is tier two doctor availability that has the greatest effect on patient flow, and with regards to consultant reviews this is more significantly determined by logistics of patient prioritisation. Although the proportion of patients being seen by a consultant within the recommended 14 hours has improved drastically since introduction of the consultant on-call extension, the majority of all paediatric patients are seen, treated and discharged without consultant input $(65.6 \%$ over cycles 1-4). This finding highlights that patient flow is dependent primarily on adequate tier two doctor cover.

\section{Limitations}

Our data were limited by the significant number of cases that had incomplete documentation (only 177 out of the 288 data sets gathered were complete), including $14 \%$ and $17 \%$ of cases where time to tier two doctor and consultant review was not documented. The percentage of complete data sets varied between each cycle (range: $46.9 \%$ to $76.6 \%$ ) but did not show any trend over time. This may have had an unknown skew on the data, and as such future cycles could seek to improve proportions of proformas that are filled completely through adjustments to the current working proforma. We also note that inaccuracies in record-keeping may have skewed results; the data collected relied on correct recording of start of consultations, which may have been incorrect with retrospective documenting.

The PDSA cycles thus far focused on tier two doctor and consultant rosters, although have not yet explored the effect of tier one doctor rosters on flow. Indeed, we note the increase in time to tier one review between cycles 3 and 4 (63-82.9 min), which future cycles aim to analyse with respect to tier one doctor rosters and total numbers of doctors of all grades per shift.

A further limitation was the multivariate factors involved in time to discharge, including nature of conditions presenting (affecting time involved in management and observation), time to implementation of plans, ward commitments of doctors and bed availability. Factors not considered which we aim to investigate in further cycles include the presence of senior availability including consultant cover in the emergency department prior to PAU admission, the number of emergency calls during a shift attended by paediatric doctors, and nursing and auxiliary staffing levels. Additionally, data on category and severity of paediatric presentations were not collected. Seasonal variations in paediatric presentations ${ }^{67}$ can cause varying numbers of acutely unwell patients including fluctuating incidences of respiratory viruses, with variable proportions of these patients between the cycles conducted in time periods in summer, spring and autumn; these patients are labour and time intensive as they require immediate stabilisation as well as extensive medical intervention. These patients can monopolise limited healthcare resources, delaying reviews of other, more stable patients, and therefore paediatric patient flow as a whole.

Our data were limited by the nature of retrospective analysis of patient notes that could not compare the multiple factors in patient management that may have skewed discharge times even with equivocal rates of tier one, two and three doctor cover.

The surprising and statistically significant increase in the average length of time a paediatric patient spends in PAU (from $229 \mathrm{~min}$ in baseline measurement to $329 \mathrm{~min}$ in cycle 4 ), despite reduced numbers of paediatric patients attending the service (329 in baseline measurement, 98 in cycle 4) is perhaps due to alternative factors which were not analysed in this quality improvement project. These may be shortfalls in nursing cover, and delays in plan implementation. Further evaluation of the factors behind this is warranted, including analysis of ward commitments of juniors.

\section{CONCLUSIONS}

In addition, our results demonstrate the significance of tier two doctor presence on paediatric flow. Tier two doctor staffing is critical in maintaining prompt paediatric reviews and therefore flow within an emergency care setting. As such, future workforce planning needs to consider and protect paediatric trainee numbers given these findings. 
Improving patient flow is particularly pertinent given the need for social distancing during the COVID-19 pandemic. Future studies would need to assess alternative factors which may delay patient flow, such as nursing, auxiliary and junior doctor staffing levels, emergency call rates during shifts, seasonal variation in presentations to PAU as well as qualitative factors such as staff morale and patient satisfaction.

Our quality improvement project provides an empirical framework to assess the impact of and priorities in rostering and logistical changes in a Paediatric Admissions Unit in response to the Facing The Future guidelines.

Contributors All authors made substantial contributions to the conception and design of this work. We have no competing interests to declare. The author responsible as guarantor is Dr. Danning Li.

Funding The authors have not declared a specific grant for this research from any funding agency in the public, commercial or not-for-profit sectors.

Competing interests None declared.

Patient and public involvement Patients and/or the public were not involved in the design, or conduct, or reporting, or dissemination plans of this research.

Patient consent for publication Not required.

Ethics approval This audit was deemed exempt from ethics committee evaluation. Audit reference for Birmingham Heartlands Hospital audit portal is 5289.

Provenance and peer review Not commissioned; externally peer reviewed.

Data availability statement Data are available on request.
Open access This is an open access article distributed in accordance with the Creative Commons Attribution Non Commercial (CC BY-NC 4.0) license, which permits others to distribute, remix, adapt, build upon this work non-commercially, and license their derivative works on different terms, provided the original work is properly cited, appropriate credit is given, any changes made indicated, and the use is non-commercial. See: http://creativecommons.org/licenses/by-nc/4.0/.

\section{REFERENCES}

1 Paling S, Lambert J, Clouting J, et al. Waiting times in emergency departments: exploring the factors associated with longer patient waits for emergency care in England using routinely collected daily data. Emerg Med J 2020;37:emermed-2019-208849.

2 RCPCH.ac.uk. Facing the future: standards for acute General paediatric services, 2015. Available: https://www.rcpch.ac.uk/sites/ default/files/2018-03/facing_the_future_standards_for_acute_general_ paediatric_services.pdf [Accessed 29 Nov 2020].

3 RCPCH.ac.uk. Facing the future audit 2017, 2017. Available: https:// www.rcpch.ac.uk/sites/default/files/2018-04/Facing\%20the\% 20Future\%20audit\%202017\%20-\%20report\%20\%28April\%202018\% 29.pdf [Accessed 29 Nov 2020].

4 Kendrew-Jones T, Farquharson S, Stewart E. G112 (P) are we facing the future? a review of acute General paediatric admissions at a regional paediatric Hospital. Arch Dis Child 2020;105.

5 Ramsden L, McColgan MP, Rossor T, et al. Paediatric outcomes and timing of admission. Arch Dis Child 2018;103:611-7.

6 Takase M, Carlin J. Modelling seasonal variations in presentations at a paediatric emergency department. Hiroshima J Med Sci 2012;61:51-8.

7 D'Souza RM, Bambrick HJ, Kjellstrom TE, et al. Seasonal variation in acute hospital admissions and emergency room presentations among children in the Australian Capital Territory. J Paediatr Child Health 2007;43:359-65 